\title{
Waveguide-based machine readable fluorescence security feature for border control and security applications.
}

JOHNY, J., BHAVSAR, K., OFFICER, S., ADAMS, M., PRABHU, R. personal use only. Systematic reproduction and distribution, duplication of any material in this paper for a fee or for commercial purposes, or modification of the content of the paper are prohibited. 


\title{
Waveguide based machine readable fluorescence security feature for border control and security applications
}

\author{
Jincy Johny ${ }^{1}$, Kaushalkumar Bhavsar ${ }^{1}$, Simon Officer², Morgan Adams ${ }^{1}$, Radhakrishna Prabhu ${ }^{1}$ \\ ${ }^{1}$ School of Engineering, Robert Gordon University, Aberdeen, United Kingdom \\ ${ }^{2}$ School of Pharmacy and Life Sciences, Robert Gordon University, Aberdeen, United Kingdom
}

\begin{abstract}
Border security challenges and immigration issues are increasing considerably in recent years. Counterfeiting and fraudulent use of identity and other travel documents are posing serious threats and safety concerns worldwide, ever since the advancement of computers, photocopiers, printers and scanners. Considering the current scenario of illegal migration and terrorism across the world, advanced technologies and improved security features are essential to enhance border security and to enable smooth transits. In this paper, we present a novel dual waveguide based invisible fluorescence security feature and a simple validation system to elevate and strengthen the security at border controls. The validation system consists of an LED (light emitting diode) as excitation source and an array photodetector which helps in the simultaneous detection of multiple features from the fluorescence waveguides. The fluorescence waveguides can be embedded into the identity document as micro-threads or tags which are invisible to the naked eye and are only machine readable. In order to improve the sensitivity, rare earth fluorescence materials are used which absorb only specific ultraviolet (UV) or visible (VIS) wavelengths to create corresponding fluorescent emission lines in the visible or infrared wavelengths. Herein, we present the preliminary results based on the fluorescence spectroscopic studies carried out on the fabricated rare earth doped waveguides. The effect of different rare earth concentrations and excitation wavelengths on the fluorescence intensity were investigated.
\end{abstract}

Keywords: Fluorescence, Rare earth, Waveguide, Spectroscopy, Security feature, Border control, Anti-counterfeiting

\section{INTRODUCTION}

In recent years, there is a considerable increase in the traveler volume and the number of international crimes reported. The border and port security agencies are in-charge of keeping their nation safe and secure from terrorists and other illegal entries. This in turn puts pressure on the border security officials to improve their security protocols and systems to prevent the fraudulent use of travel documents like passports [1-2] and other identity documents. The threats posed by fraudsters are increasing day by day creating serious concerns worldwide. Numerous technological developments have emerged for enhancing the security features, such as electronic passports (e-passports) [3-4] or the electronic machine-readable travel document (e-MRTD) [5] which contains an RFID (Radio Frequency Identification) tag [6] or an electronic chip [7], biometrics with the individual's facial image and fingerprints [8-9]. However, the fraudsters still use different techniques including counterfeiting [10], cloning [11], tampering, alteration, reusing, stealing, misusing of genuine documents belonging to a similar looking person for creating fake documents [12]. Computer revolution and the rapid developments in the field of photocopiers, printers and scanners have made counterfeiting and creation of fake documents much simpler. Hence, it is becoming harder to track down these counterfeiters because of their rapid adoption and adaptation of the leading technologies [13]. Hence, new innovative technologies with highly advanced security features are needed to fight against these fraudulent attacks and to ensure safe and efficient passage of travelers across international borders.

Luminescent or fluorescent based security technique is an emerging area and a promising approach for securing valuable printed documents from counterfeiting and other fraud recreations. One of the main attractions of a luminescent security feature is that it adds authenticity to the printed document [14]. Now-a-days, fluorescent dye materials are widely used for labelling and creating visible and invisible indicia or markings on the documents, which becomes visible only by the application of irradiation such as ultraviolet (UV) light [15]. Although the fluorescent dye based labelling technique is difficult to counterfeit, once the particular dye component is identified by the fraudster it becomes fairly easier to reproduce 
them. Hence, new ink materials based on rare earth luminescent nanomaterials such as europium (Eu), thulium (Tm), erbium (Er), ytterbium ( $\mathrm{Yb}$ ), dysprosium (Dy) and terbium $(\mathrm{Tb})$ doped within suitable hosts are being developed for the secure labelling of printing products such as passports, visa documents and banknotes [16]. The rare earth doped waveguide can easily be incorporated into paper products by adding these rare earth micro-fibres into the cellulose fibre suspension used for paper making. Following this, papers with the rare earth waveguide security feature can be easily synthesized, through normal sieving and drying process of the obtained sheets of interwoven fibres from the suspension [17]. The main advantage of a rare earth based optical security feature is that they have specific fluorescent emission peaks which are dependent on the incident light or the excitation wavelength. Furthermore, these rare earth waveguide security features provide a color or spectral differentiation which aids in quickly identifying the fake document, while authenticating the genuine ones [16]. Moreover, rare earth materials have narrow fluorescent emission peaks, compared to inks and dyes which has a broader emission spectrum, enabling scope for multiple rare earth security feature on the same space [18]. Hence, rare earth fluorescent materials are promising candidates for securing valuable documents against counterfeiting issues, because they are difficult to replicate but easier to authenticate using simple fluorescence spectroscopic techniques [18]. The unique characteristics and features of rare earth optical materials are encouraging research in this direction [1921].

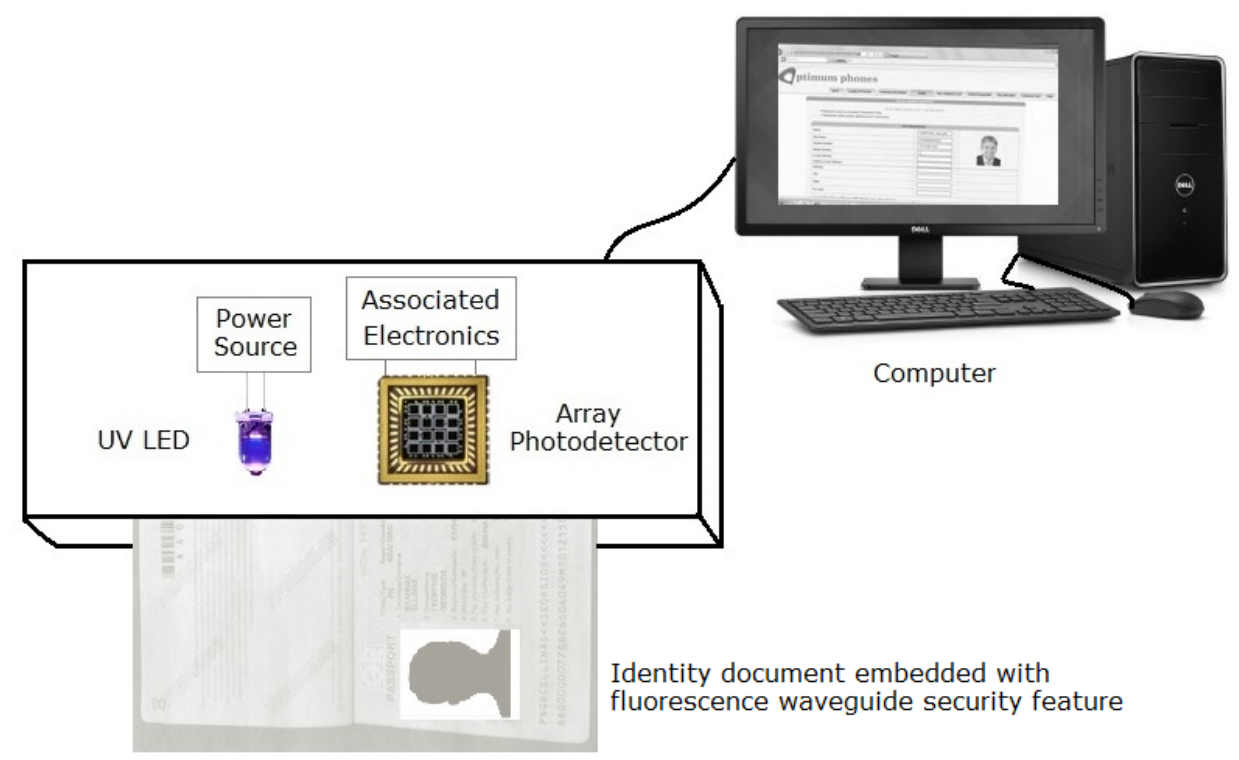

Figure 1. A schematic view of the fluorescence waveguide security feature validation system

In this paper, we present a dual waveguide-based fluorescence security feature and a validation system to enhance security at border controls. Figure 1 shows the schematic view of the simple validation system, comprising of an LED and an array photodetector for the detection of multiple security features from the rare earth doped fluorescence waveguides embedded into the travel document. The photodiode array helps in detecting multiple fluorescent signatures from the doped waveguide as it can sense multiple wavelengths covering a broader range [22]. The main attraction of this validation system is that it is very simple and cost-effective. Moreover, the identity document can be easily verified using the fluorescence security feature authentication technique. The speciality of rare earth fluorescent materials is that they absorb only specific UV or visible wavelengths and produce corresponding fluorescent emissions in the visible or IR wavelengths. The main advantage of rare earth fluorescence waveguides is that they can be easily incorporated into the travel documents like passports and visa cards as micro-threads or tags which are only machine readable and not visible to the naked eye. Besides this, the waveguide security feature facilitates unique light wave guidance, which means light can be irradiated at one end of the waveguide and the guided fluorescence signal can be collected from its other end. Implementation of these new and advanced machine-readable security features helps in stopping fake identities from crossing the borders, whilst facilitating the fast, safe, and smooth crossing for common travelers. Furthermore, this waveguide based fluorescent technology can easily work along with other existing security features, serving as an additional security feature within the document. To sum up, the new technique will speed up border control procedures and will overcome the current hassle of multiple manual identification checks. In the present work different rare earth ( $\mathrm{Tb}$ and $\mathrm{Dy}$ ) doped waveguides were obtained by using the fibre drawing technique. The rare earth elements were doped in the borosilicate glass (BSG) host matrix to fabricate waveguides of different concentrations [23]. Herein, we report the preliminary results obtained based on the fluorescence 
spectroscopic studies carried out on the fabricated rare earth doped waveguide. A detailed study was carried out on the effect of rare earth concentrations and excitation wavelengths on the fluorescence intensity.

\section{METHODOLOGY}

\subsection{Working Principle}

The waveguide security feature is based on the unique fluorescence signatures of the rare earth doped materials. Fluorescence is actually the light emission caused when a material is irradiated with light (normally ultraviolet or visible light). Typically, fluorescence emission occurs within a nanosecond to millisecond time span after light irradiation. It involves the excitation of electrons from a lower energy state into a higher energy state, from where radiative decay is possible. Normally, the emitted wavelengths are longer than the excitation wavelengths [24]. The emitted fluorescence from the rare earth doped waveguides can be detected using an array photodetector and used as the security feature. Important advantages of the rare earth waveguide security feature include: unique excitation-emission patterns, narrow fluorescence emissions, wavelength differentiation, wave guidance and are invisible and machine readable.

\subsection{Waveguide Preparation}

The fluorescent waveguide is based on Tb and Dy doped borosilicate glass fabricated though the fibre drawing process. The starting materials used for the preparation of different concentrations of $\mathrm{Tb}$ and Dy doped borosilicate glass waveguides were zinc oxide $(\mathrm{ZnO})$, boron oxide $\left(\mathrm{B}_{2} \mathrm{O}_{3}\right)$, silicon dioxide $\left(\mathrm{SiO}_{2}\right)$, terbium III chloride hexahydrate $\left(\mathrm{TbCl}_{3} \cdot 6 \mathrm{H}_{2} \mathrm{O}\right)$ and dysprosium III chloride hexahydrate $\left(\mathrm{DyCl}_{3} \cdot 6 \mathrm{H}_{2} \mathrm{O}\right)$. All chemicals were purchased from Sigma Aldrich with greater than $99 \%$ purity.

The batch mixture of zinc borosilicate glass was:

$$
60 \mathrm{ZnO}+20 \mathrm{~B}_{2} \mathrm{O}_{3}+20 \mathrm{SiO}_{2}(\mathrm{~mol} \%)
$$

The weighed chemical batches consist of $4.5717 \mathrm{~g} \mathrm{ZnO}, 1.3034 \mathrm{~g} \mathrm{~B}_{2} \mathrm{O}_{3}$ and $1.1253 \mathrm{~g} \mathrm{SiO}_{2}$, giving a total weight of $7 \mathrm{~g}$.

The rare earth doped borosilicate glass batch mixture was:

$$
(60-x) \mathrm{ZnO}+20 \mathrm{~B}_{2} \mathrm{O}_{3}+20 \mathrm{SiO}_{2}+x \mathrm{La}^{3+} \text { (where } \mathrm{La}^{3+}=\mathrm{Tb} \text { and Dy) }
$$

Where $x$ value relates to the decrease in $\mathrm{ZnO}$ composition and the corresponding rare earth concentration increase from $0.5,1.0,1.5$, and $2.0 \mathrm{~mol} \%$ [25].

All the batches each of $7 \mathrm{~g}$ were ground on an agate ball mill for 7 minutes. After the ball milling process the homogenous powder mixture was transferred into a platinum crucible and was introduced into the Carbolite 1400 high temperature furnace at a starting set temperature of $550{ }^{\circ} \mathrm{C}$. After 30 minutes the temperature was increased to $900{ }^{\circ} \mathrm{C}$ and maintained for an hour. Following this the temperature was increased to 1000,1100 and $1200{ }^{\circ} \mathrm{C}$, for one hour at each stage. Finally, at $1200{ }^{\circ} \mathrm{C}$ the fully melted powdered chemicals forming the glass melt was poured onto a brass plate to obtain the fibre preform. Subsequently, the rare earth doped glass preform was pulled into micron sized waveguides.

\subsection{Waveguide Characterization}

The fabricated rare earth doped waveguides were characterized using the Edinburgh Instrument's fluorescent spectrometer (F900). Excitation and emission scans were run on the glass samples to obtain their excitation peak wavelengths and their corresponding fluorescent signatures. For the imaging and size determination of the waveguides, a Zeiss EVO LS10 scanning electron microscope (SEM) was used.

The experimental setup used to characterize the dual waveguide configuration embedded into the identity document consisted of UV LED and UV-VIS spectrometer. The LED purchased from Nichia Corporation with peak wavelength, $\lambda_{P}=385 \mathrm{~nm}$ and full spectral width at half maximum, FWHM $=15 \mathrm{~nm}$ was used as the light source. The UV irradiation was coupled into the Tb-Dy dual waveguide through a lens assembly and the fluorescence signals were detected from its other end using the StellarNet (EPP2000C) spectrometer.

\section{RESULTS AND DISCUSSIONS}

The fluorescence characteristics of the micron sized rare earth doped borosilicate glass waveguide fabricated using the fibre drawing method was validated. Figure 2(a) shows the image of a portion of the prepared sample passport bio-data 
page embedded with Tb doped waveguide security feature. In figure 2(b), we can observe green fluorescence emission from the Tb doped waveguide $\left(\lambda_{e m}=544 \mathrm{~nm}\right)$ incorporated into the passport page, when irradiated using a UV LED of peak wavelength, $\lambda_{p}=385 \mathrm{~nm}$. Figure 3 shows the SEM images corresponding to the cross-sectional and lateral view of the developed rare earth doped waveguide. From the SEM images, the circular waveguide diameter was measured to be around $50 \mu \mathrm{m}$.

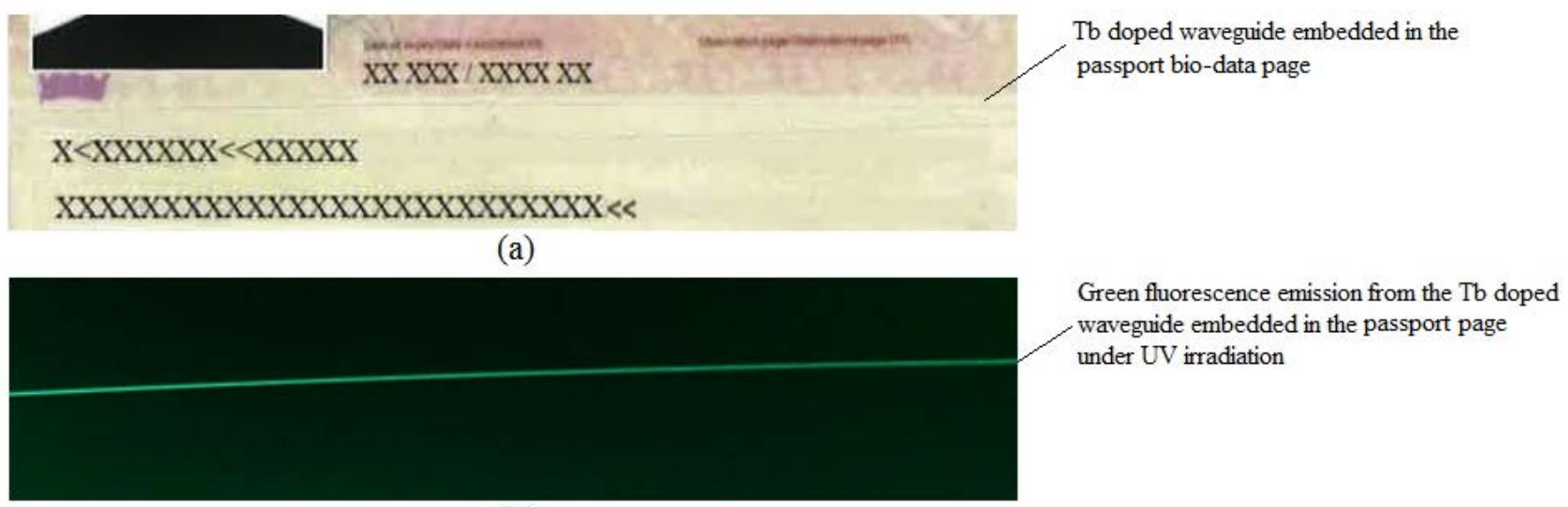

(b)

Figure 2. (a) A part of passport bio-data page embedded with Tb doped waveguide security feature in ambient light conditions and (b) green fluorescence emission from $\mathrm{Tb}$ doped waveguide embedded in the passport page when irradiated using UV LED in a dark room

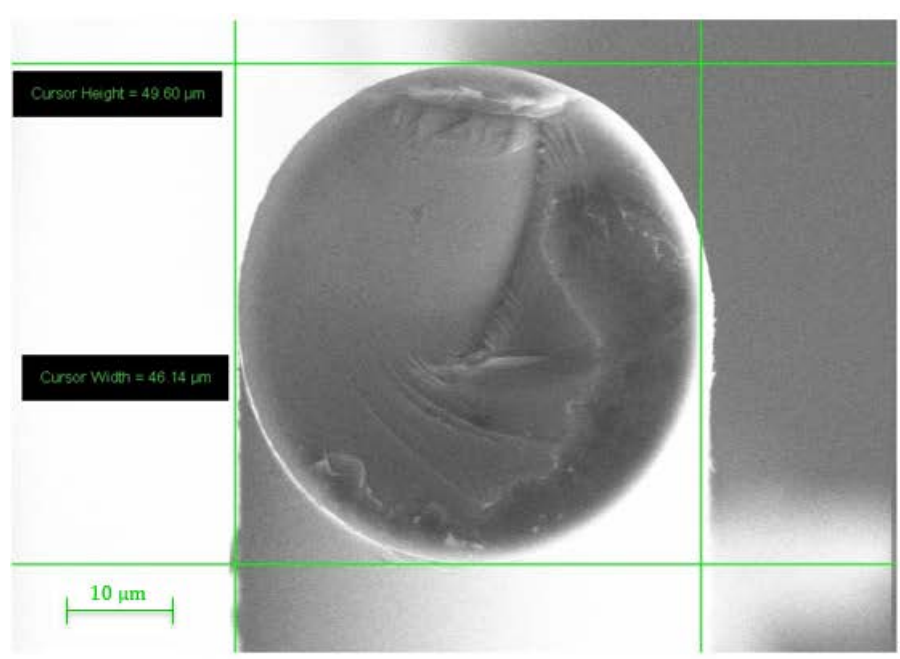

(a)
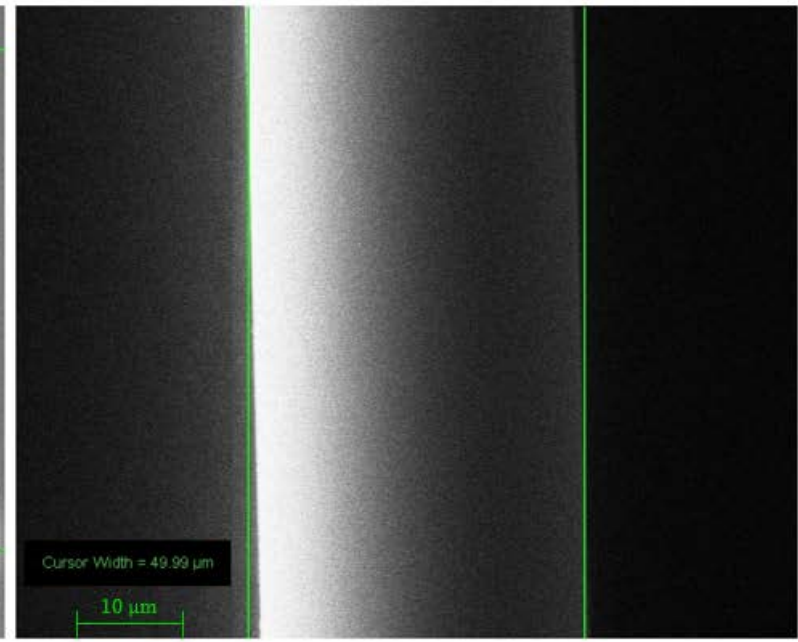

(b)

Figure 3. SEM images showing the (a) cross-section and (b) lateral view of the rare earth doped waveguide

Spectral characterization of the Tb-Dy (both $1.5 \mathrm{~mol} \%$ concentration) dual waveguide configuration embedded into the identity document was validated using the experimental setup explained in section 2.3. Figure 4 shows the output spectrum obtained from the dual waveguide when irradiated using $385 \mathrm{~nm}$ UV LED. $385 \mathrm{~nm}$ LED was chosen because, it was identified that both $\mathrm{Tb}$ and Dy samples had highest excitation peaks in this range. As we can observe from the graph, the peak in the $385 \mathrm{~nm}$ region corresponds to the LED excitation peak and the peak around $544 \mathrm{~nm}$ and $575 \mathrm{~nm}$ corresponds to the $\mathrm{Tb}$ and Dy signature peaks. The peak around $485 \mathrm{~nm}$ is present in both $\mathrm{Tb}$ and Dy samples. These initial results show scope for further improvement and a strong potential for multi-waveguide security feature. 


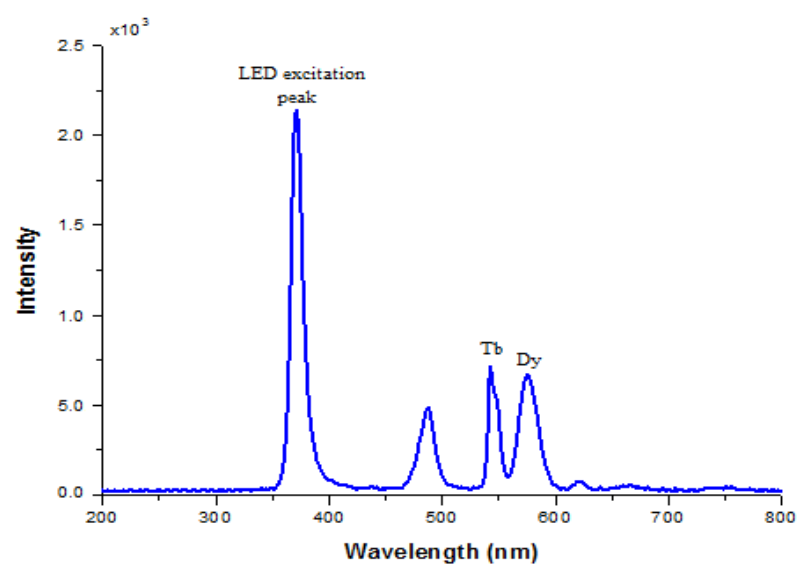

Figure 4. Output spectrum of the 1.5 mol\% Tb-Dy dual waveguide

\subsection{Characteristic features of rare earth doped materials}

In order to characterize the features of rare earth doped materials, fluorescence spectroscopic studies were carried out on the glass samples using the Edinburgh instruments fluorescence spectrometer. Figure 5 shows the measured excitation spectra of different concentrations of Tb doped BSG recorded in the wavelength range $300-525 \mathrm{~nm}$, corresponding to the green fluorescent emission at $544 \mathrm{~nm}$. In the graphs Tb05, Tb10, Tb15 and Tb20 represent the glass codes for the 0.5, 1, 1.5 and 2 mol\% Tb doped BSG. The excitation spectrum displayed peaks at 304, 318, 354, 376, 406 and $486 \mathrm{~nm}$. However, the bands at 304 and $406 \mathrm{~nm}$ were weaker compared to other excitation peaks. The most intense peak at $376 \mathrm{~nm}$ corresponds to the transition from the Tb ground state ${ }^{7} \mathrm{~F}_{6}$ to the higher excited level $\left({ }^{5} \mathrm{D}_{3},{ }^{5} \mathrm{G}_{6}\right)$ [26] suggests the suitability of UV LEDs for irradiating the Tb doped waveguide and obtaining its corresponding fluorescent emission.

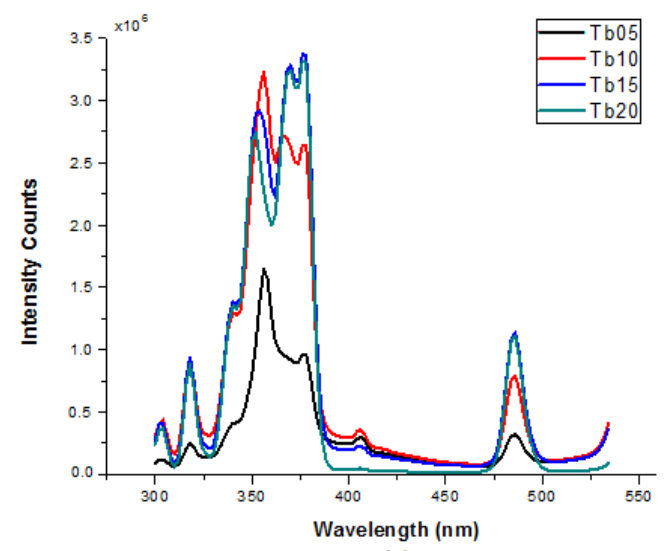

(a)

Figure 5. Excitation Spectra for different Tb concentrations corresponding to $544 \mathrm{~nm}$ fluorescent emission

Figure 6(a) depicts the optical fluorescence spectra of the Tb doped glass in response to an optical excitation of $376 \mathrm{~nm}$. This analysis was carried out in order to understand the effect of terbium concentration on its fluorescent intensity and thereby optimise the terbium doping concentration level within the waveguide. The terbium characteristic fluorescence peak was obtained at $544 \mathrm{~nm}$ corresponding to its green emission. The intensity variation of $544 \mathrm{~nm}$ Tb fluorescence as a function of its concentration, for $376 \mathrm{~nm}$ excitation, is shown in figure 6(b). From the graph, we can observe that the Tb doped borosilicate glass exhibits a strong luminescence at $1.5 \mathrm{~mol} \%$ concentration. Further increase of the rare earth concentration to $2 \mathrm{~mol} \%$ did not improve the fluorescence signal intensity. Hence, $1.5 \mathrm{~mol} \%$ is the optimum doping concentration for the earth doped waveguide to obtain maximum signal intensity. 


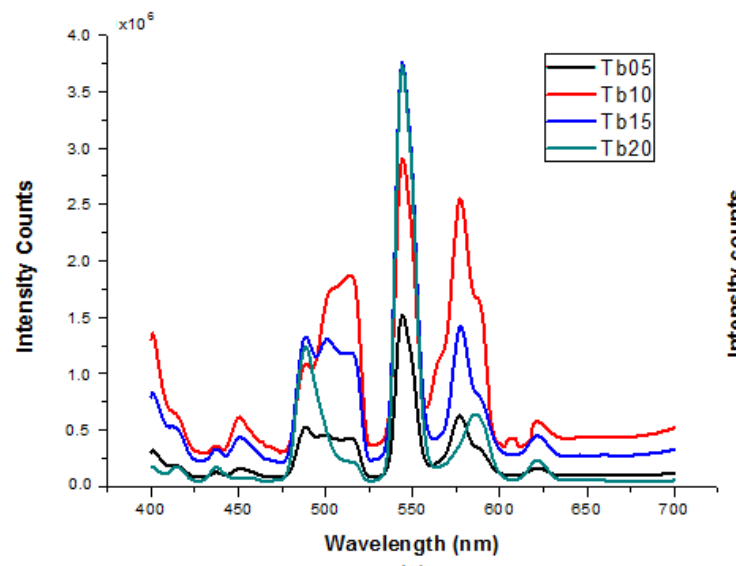

(a)

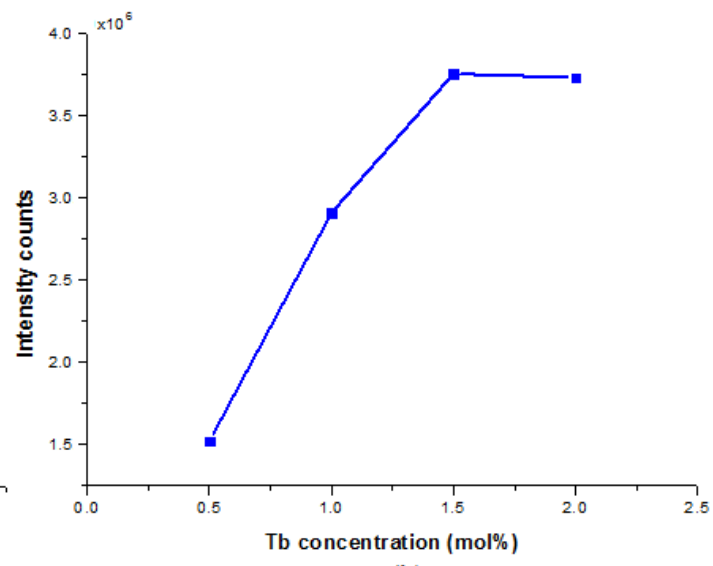

(b)

Figure 6. (a) Emission spectra for different $\mathrm{Tb}$ concentrations corresponding to $376 \mathrm{~nm}$ excitation and (b) fluorescent intensity vs $\mathrm{Tb}$ concentration for $544 \mathrm{~nm}$ emission under $376 \mathrm{~nm}$ excitation

Besides this, to analyze the effect of different excitation wavelengths on the fluorescence intensity of the Tb doped BSG, the emission scan was recorded for different excitation wavelengths as depicted in figure 7 (a). In figure 7(b), the variation in fluorescent intensity is plotted as a function of excitation wavelengths for the $544 \mathrm{~nm} \mathrm{~Tb}$ emission. For the different excitation wavelengths tried, highest fluorescence intensity was obtained for $376 \mathrm{~nm}$ excitation followed by 354 $\mathrm{nm}, 486 \mathrm{~nm}$ and $318 \mathrm{~nm}$.

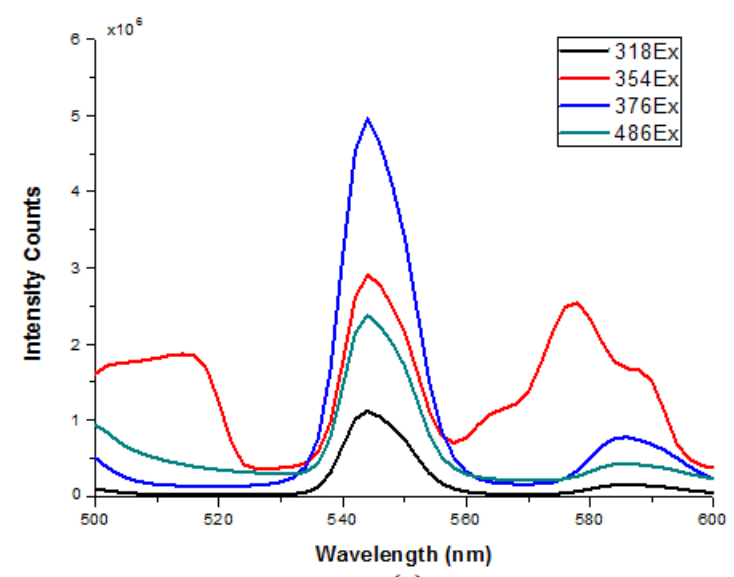

(a)

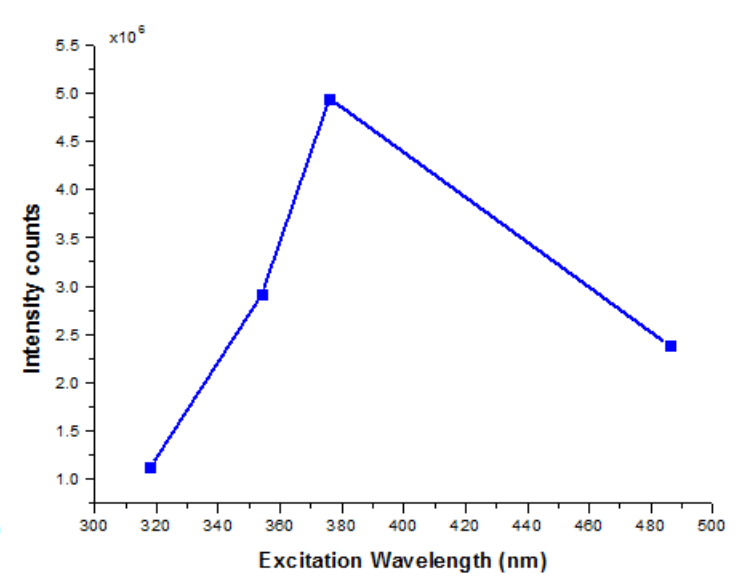

(b)

Figure 7. (a) Emission spectra of $1.5 \mathrm{~mol} \% \mathrm{~Tb}$ for different excitation wavelengths and (b) intensity variation of $1.5 \mathrm{~mol} \% \mathrm{~Tb}$ for different excitation wavelengths

After characterizing Tb doped glass samples, similar excitation-emission studies were carried out on Dy doped samples. Figure 8 shows the excitation spectra obtained for different concentrations of Dy doped BSG in the spectral range $300-500 \mathrm{~nm}$, corresponding to the $575 \mathrm{~nm}$ fluorescent emission. Dy05, Dy10, Dy15 and Dy20 represent the glass codes for $0.5,1,1.5$ and $2 \mathrm{~mol} \%$ Dy doped BSG. The excitation spectrum shows different peaks at 350, 364, 388, 424, 452 and $470 \mathrm{~nm}$ among which the $388 \mathrm{~nm}$ peak that corresponds to the transition from the Dy ground state $\left({ }^{6} \mathrm{H}_{15 / 2}\right)$ to the higher energy level $\left({ }^{4} \mathrm{I}_{13 / 2},{ }^{4} \mathrm{~F}_{7 / 2}\right)$ [27], has the highest intensity, hence the same UV LED can be used for illuminating the Dy doped waveguide to obtain its respective fluorescent emission. 


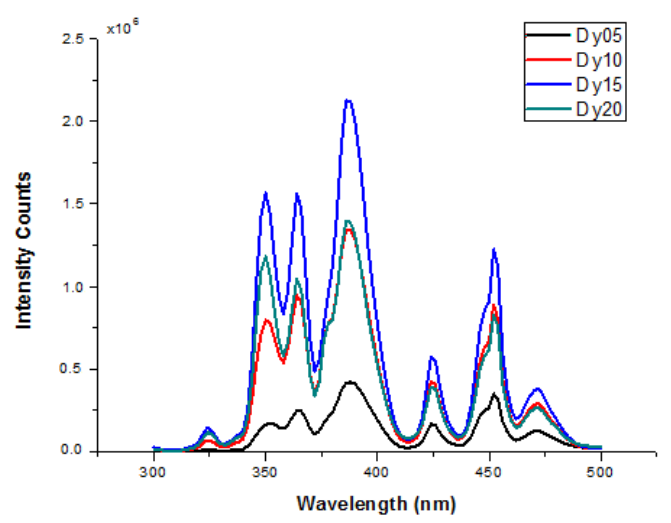

Figure 8. Excitation Spectra for different Dy concentrations corresponding to $575 \mathrm{~nm}$ fluorescent emission

Figure 9(a) shows the fluorescent emission spectra of Dy doped borosilicate glass for $388 \mathrm{~nm}$ excitation. The dysprosium signature peak was obtained around $575 \mathrm{~nm}$ corresponding to its yellowish fluorescence emission. The intensity variation of $575 \mathrm{~nm}$ Dy fluorescence as a function of its concentration, is shown in figure 9(b), when the glass sample was excited at $388 \mathrm{~nm}$. From the graph it is very clear that the Dy doped BSG exhibits a strong luminescence at $1.5 \mathrm{~mol} \%$ concentration and further increase of Dy concentration did not improve the fluorescence signal. Hence, $1.5 \mathrm{~mol} \%$ was the optimum doping concentration for the Dy doped waveguide.

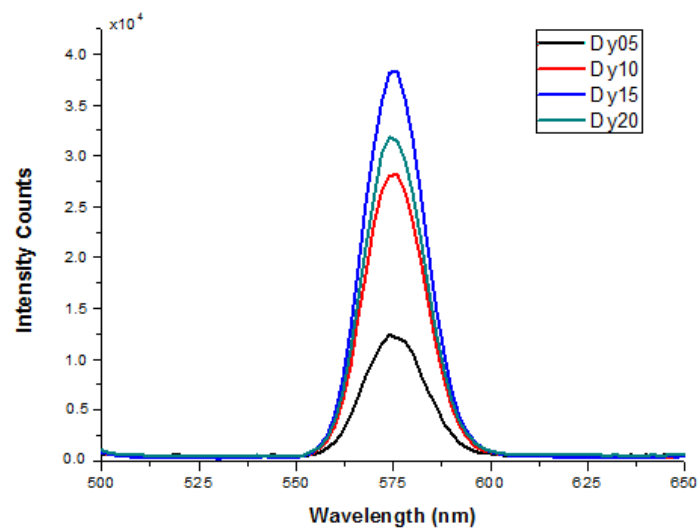

(a)

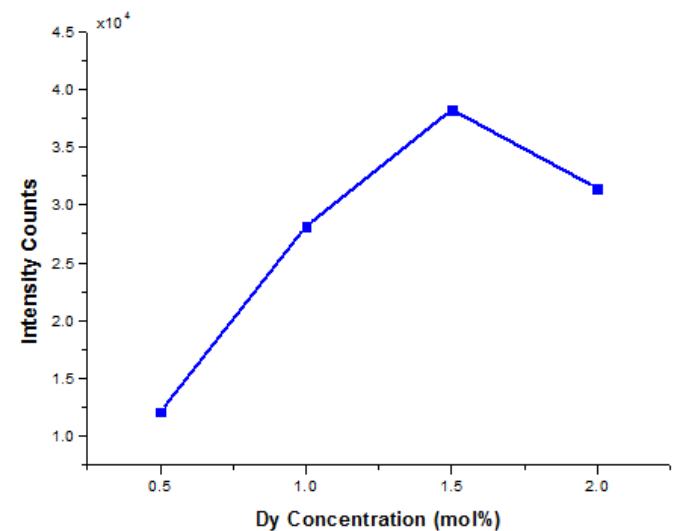

(b)

Figure 9. (a) Emission spectra for different Dy concentrations corresponding to $388 \mathrm{~nm}$ excitation and (b) fluorescent intensity vs Dy concentration for $575 \mathrm{~nm}$ emission under $388 \mathrm{~nm}$ excitation

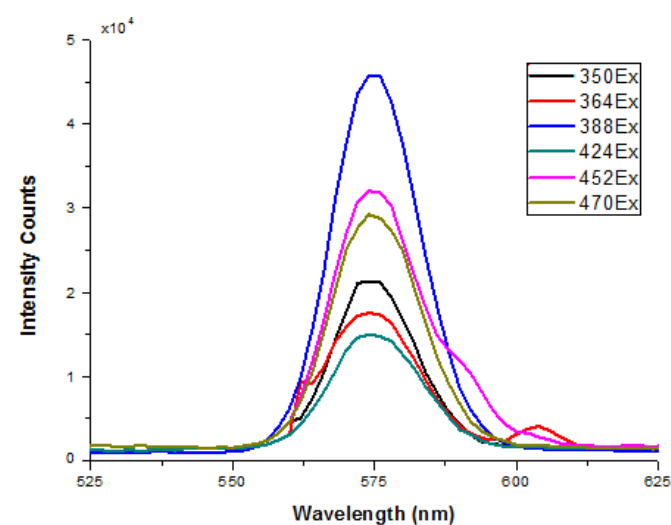

(a)

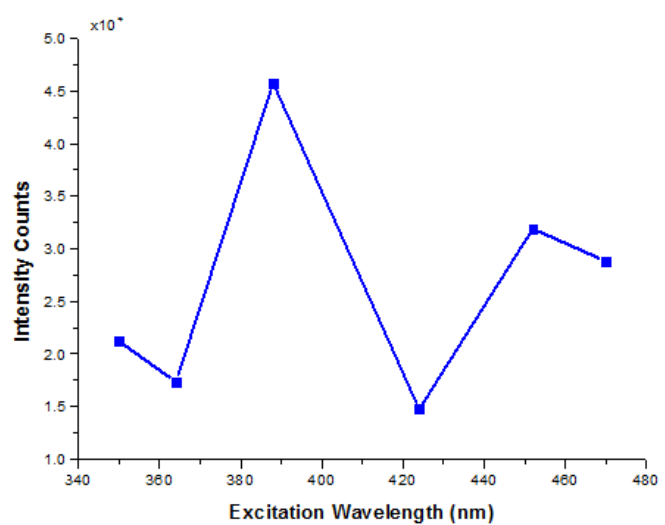

(b)

Figure 10. (a) Emission Spectra of $1.5 \mathrm{~mol} \%$ Dy for different excitation wavelengths and (b) Intensity variation of $575 \mathrm{~nm}$ emissions for different Dy concentrations under $452 \mathrm{~nm}$ excitation 
Furthermore, to analyze the effect of different excitation wavelengths on the fluorescence intensity of Dy doped BSG waveguide, the emission scan was recorded for different excitation wavelengths as depicted in figure 10 (a). In figure 10(b), the respective variation in fluorescent intensity is plotted as a function of excitation wavelengths corresponding to the $575 \mathrm{~nm}$ Dy emission. For the 6 excitation wavelengths tried, highest fluorescence intensity was obtained for $388 \mathrm{~nm}$ excitation followed by 452, 470, 350, 364 and $424 \mathrm{~nm}$.

The spectroscopic studies carried out on the fabricated rare earth doped waveguide identified the optimum rare earth concentration and the appropriate excitation wavelength for the Tb and Dy waveguides. UV LEDs were found suitable for the validation system as the excitation source. One main advantage is that, the UV LEDs can be used for the irradiation or excitation of both the rare earth elements ( $\mathrm{Tb}$ and $\mathrm{Dy}$ ) simultaneously and the output fluorescence signal would still be distinguishable since the emission wavelengths are discrete.

\section{CONCLUSION}

A novel rare earth doped dual waveguide fluorescence security feature and a simple validation system consisting of an LED and an array photodetector is proposed to enhance the security at border controls. The rare earth waveguide security feature can be easily embedded within the travel documents as micron size threads or tags. These invisible fluorescent security features, when irradiated with light, generates specific fluorescent emissions which are unique for each rare earth element. These specific rare earth fluorescence signals are not visible to the naked eye and are only machine readable. Multiple rare earth elements doped into the waveguide provides advanced security features along with high level of authenticity to these identity documents. In addition to improvement of border security control, it facilitates smoother and faster traveler clearances and transits at the ports. Another important advantage of this security feature is that it cannot be easily duplicated using any other reproduction technologies. Furthermore, this new waveguide fluorescent technique can work in tandem with other existing security features within the document, serving as an additional security feature. The proposed security feature for the travel document is a very simple, cost effect and robust technique. Fluorescence spectroscopic characterization of the fabricated rare earth doped waveguide was carried out to analyze the effect of different excitation wavelengths and rare earth concentrations on the output fluorescence signal intensity. The optimum rare earth concentration for the dual waveguide configuration and the appropriate LED excitation source was identified for the validation system. Implementation of these new and advanced machine-readable security features helps in stopping fraudsters from crossing international borders, whereas facilitating fast and safe crossing for genuine travelers.

\section{REFERENCES}

[1] Salter MB. Passports, Mobility, and Security: How smart can the border be? International Studies Perspectives. 2004; 5(1):71-91.

[2] Lehtonen P, Aalto P. Smart and secure borders through automated border control systems in the EU? The views of political stakeholders in the Member States. European Security. 2017; 26(2):207-225.

[3] Juels A, Molnar D and Wagner D. Security and Privacy Issues in E-passports. Security and Privacy for Emerging Areas in Communications Networks, 2005. Secure Comm 2005. First International Conference on IEEE; 2005. 74-88.

[4] Schouten B, Jacobs B. Biometrics and their use in e-passports. Image and Vision Computing. 2009; 27(3):305312.

[5] Mayes KE, Markantonakis K. Smart cards, tokens, security and applications.: Springer; 2008.

[6] Koscher K, Juels A, Brajkovic V and Kohno T. EPC RFID tag security weaknesses and defenses: passport cards, enhanced drivers licenses, and beyond. Proceedings of the 16th ACM conference on Computer and communications security ACM; 2009. 33-42.

[7] Rana A, Sportiello L. Implementation of security and privacy in ePassports and the extended access control infrastructure. International Journal of Critical Infrastructure Protection. 2014; 7(4):233-243.

[8] Prabhakar S, Pankanti S, Jain AK. Biometric recognition: Security and privacy concerns. IEEE security \& privacy. 2003; (2):33-42.

[9] Del Rio JS, Moctezuma D, Conde C, de Diego IM, Cabello E. Automated border control e-gates and facial recognition systems. Computers \& Security. 2016; 62:49-72.

[10] Lowe P. Counterfeiting: links to organised crime and terrorist funding. Journal of Financial Crime. 2006; 13(2):255-257. 
[11] Calderoni L, Maio D. Cloning and tampering threats in e-Passports. Expert Systems with Applications. 2014; 41(11):5066-5070.

[12] Rudner M. Misuse of passports: identity fraud, the propensity to travel, and international terrorism. Studies in Conflict \& Terrorism. 2008; 31(2):95-110.

[13] Ambadiyil S, Krishnendu P, Pillai VM and Prabhu R. Banknote authentication using chaotic elements technology. Banknote authentication using chaotic elements technology. Counterterrorism, Crime Fighting, Forensics, and Surveillance Technologies, International Society for Optics and Photonics; 2017. 1044104.

[14] Kaule W, Schwenk G, Stenzel G, inventors; Giesecke, Devrient GmbH, assignee. Printed document having a value, with luminescent authenticity feature. United States patent US 6,506,476. 2003 Jan 14.

[15] Dukler S, Landman A, inventors; INKSURE Ltd, assignee. Security documents with visible and invisible markings. United States patent US20030035131A1. 2003 Feb 20.

[16] Officer S, Prabhu GR, Pollard P, Hunter C and Ross GA. Novel online security system based on rare-earth-doped glass microbeads. Optical Security and Counterfeit Deterrence Techniques, International Society for Optics and Photonics; 2004. 387-396.

[17] Ververis C, Georghiou K, Christodoulakis N, Santas P, Santas R. Fiber dimensions, lignin and cellulose content of various plant materials and their suitability for paper production. Industrial crops and products. 2004; 19(3):245-254.

[18] Ross GA, Pollard P, Hunter C, Officer S, Prabhu GR, inventors; NCR Corp, assignee. Optically detectable security feature. United States patent US 7,129,506. 2006 Oct 31.

[19] Goltsos W, inventor; Spectra Systems Corp, assignee. Optically-based system for processing banknotes based on security feature emissions. United States patent US 6,744,525. 2004 Jun 1.

[20] Anh TK, Loc DX, Huong TT, Vu N, Le Quoc Minh. Luminescent nanomaterials containing rare earth ions for security printing. International Journal of Nanotechnology. 2011; 8(3-5):335-346.

[21] Campos-Cuerva C, Zieba M, Sebastian V, Martínez G, Sese J, Irusta S, et al. Screen-printed nanoparticles as anticounterfeiting tags. Nanotechnology. 2016; 27(9):095702.

[22] Es'haghi Z. Photodiode array detection in clinical applications; quantitative analyte assay advantages, limitations and disadvantages. Photodiodes-Communications, Bio-Sensings, Measurements and High-Energy Physics.: InTech; 2011.

[23] Kullberg A, Lopes A, Veiga J, Lima M, Monteiro R. Formation and crystallization of zinc borosilicate glasses: Influence of the ZnO/B2O3 ratio. Journal of Non-Crystalline Solids. 2016; 441:79-85.

[24] Albrecht C. Joseph R. Lakowicz: Principles of fluorescence spectroscopy. Analytical and Bioanalytical chemistry. 2008; 390(5):1223-1224.

[25] Nkwoada A, Officer S. Novel Dysprosium and Terbium Doped Taggants for Hydrocarbon Identification. Journal of Advanced Chemical Sciences. 2016; :296-298.

[26] Sontakke AD, Biswas K, Annapurna K. Concentration-dependent luminescence of Tb3+ ions in high calcium aluminosilicate glasses. Journal of Luminescence. 2009 Nov 1;129(11):1347-55.

[27] Lakshminarayana G, Qiu J. Photoluminescence of Pr3+, Sm3+ and Dy3+-doped SiO2-Al2O3-BaF2-GdF3 glasses. Journal of Alloys and Compounds. 2009 May 12;476(1-2):470-6. 